\title{
BMJ Open Tobacco control policies and perinatal and child health: a systematic review and meta-analysis protocol
}

\author{
Jasper V Been, ${ }^{1,2,3}$ Johan P Mackenbach, ${ }^{4}$ Christopher Millett, ${ }^{5}$ Sanjay Basu, ${ }^{6}$ \\ Aziz Sheikh ${ }^{2,3,7}$
}

To cite: Been JV, Mackenbach JP, Millett C, et al. Tobacco control policies and perinatal and child health: a systematic review and meta-analysis protocol. BMJ Open 2015;5:e008398. doi:10.1136/bmjopen-2015008398

- Prepublication history and additional material is available. To view please visit the journal (http://dx.doi.org/ 10.1136/bmjopen-2015008398).

Received 3 April 2015 Revised 18 June 2015 Accepted 19 July 2015

\section{CrossMark}

For numbered affiliations see end of article.

Correspondence to Dr Jasper V Been; j.been@erasmusmc.nl

\section{ABSTRACT}

Introduction: Children experience considerable morbidity and mortality due to tobacco smoke exposure. Tobacco control policies may benefit child health by reducing this exposure. We aim to comprehensively assess the effects of the range of tobacco control policies advocated by the WHO on perinatal and child health.

Methods and analysis: We will systematically search 19 electronic literature databases (from inception) for published studies, and the WHO International Clinical Trials Registry Platform for unpublished studies. Additional work will be identified via handsearching references and citations, and through consulting an international panel of experts. No language restrictions will apply. Following Cochrane Effective Practice and Organisation of Care (EPOC) guidelines, randomised and clinical controlled trials, controlled before-and-after studies, and interrupted time series designs, are eligible. Studies of interest will assess the impact of any of the WHO-advocated tobacco control policies contained in the MPOWER acronym (except 'Monitoring tobacco use') on at least one outcome of interest among children aged $0-12$ years. The primary outcomes are: perinatal mortality, preterm birth, asthma exacerbations requiring hospital attendance and respiratory infections requiring hospital attendance. Data will be extracted using customised forms and authors will be contacted to obtain missing information. Risk of bias will be assessed using EPOC criteria. Findings will be reported in narrative and tabular form. Between-study heterogeneity will be assessed clinically and statistically using $\mathrm{I}^{2}$. If appropriate and possible, random-effects meta-analysis will be conducted for each unique combination of intervention and outcome. Subgroup analyses will be performed to assess the influence of the comprehensiveness of each policy, and to explore the impact of each policy according to socioeconomic status.

Ethics and dissemination: No ethical assessment is necessary as we will summarise existing studies. We will publish our findings in a peer-reviewed scientific journal.

Trial registration number: PROSPERO; CRD42015023448.

\section{INTRODUCTION}

Tobacco use remains the primary cause of preventable mortality worldwide. ${ }^{1}$ Unwillingly, over $40 \%$ of children suffer from the health risks associated with regular exposure to other people's tobacco smoke (secondhand smoke, SHS). ${ }^{2}$ Respiratory diseases among children account for over half of the annual 10.9 million healthy life years lost globally as a result of SHS exposure. ${ }^{2}$ In addition, tobacco smoke exposure before birth and during early life is associated with a range of adverse pregnancy outcomes including stillbirth and low birth weight, ${ }^{3}$ as well as with congenital anomalies and sudden infant death syndrome (SIDS). ${ }^{4} 5$ Governmental action to protect infants and children from the health dangers of SHS during these critical phases of development is of paramount importance, as children have no means by which to regulate their own level of exposure.

Following on from its 2003 Framework Convention on Tobacco Control (FCTC), the WHO has developed a package of six key policy recommendations summarised in the MPOWER acronym (ie, Monitor tobacco use and prevention policies; Protect people from tobacco smoke; Offer help to quit tobacco use; Warn about the dangers of tobacco; Enforce bans on tobacco advertising, promotion and sponsorship; and Raise taxes on tobacco). ${ }^{1}$ Despite progress having been made regarding the implementation of MPOWER policies in individual countries, the majority of the world's population remains unprotected by even a single tobacco control policy at the highest level. ${ }^{1}$ Given the substantial contribution of children to the global burden of disease due to SHS exposure, and their inability to influence SHS exposure, it is surprising that impact evaluations of tobacco control policies have generally excluded children, 
focusing on adult health and smoking rates. ${ }^{6-9}$ In the first systematic review on the health impact of smoke-free policies (' $\mathrm{P}$ ' in MPOWER) among children, we recently showed important reductions in preterm birth and severe asthma exacerbations, ${ }^{10}$ highlighting the considerable potential for tobacco control to benefit child health. Additional studies are now also showing positive impact on other outcomes, such as paediatric respiratory infections and perinatal mortality, and of other tobacco control policies, such as tobacco taxation, on early-life health. ${ }^{11-16} \mathrm{~A}$ comprehensive assessment of the impact of the different MPOWER policies on child health is, however, lacking.

We therefore aim to systematically investigate the impact of tobacco control policies on key perinatal and child health outcomes. This will provide an update of our recent systematic review and meta-analysis on smokefree legislation and early-life health, ${ }^{10}$ while at the same time significantly broadening the scope of this work by evaluating the child health impact of a much wider range of WHO-advocated tobacco control policies. Where possible and appropriate, we will employ meta-analysis to produce aggregate effect measures of the association between the policies and each outcome. By providing a comprehensive perspective on the health impact of a range of tobacco control policies among children, who unwillingly contribute importantly to the overall burden of SHS-associated adverse health outcomes, our findings can guide policy-making in those parts of the world where MPOWER implementation is lagging behind. We will develop policy recommendations and identify the key remaining knowledge gaps in the field, and, accordingly, propose future research priorities.

\section{METHODS}

We followed the PRISMA-P guideline for preferred reporting for systematic review and meta-analysis protocols in preparing this protocol. ${ }^{17}$ This protocol is registered with the PROSPERO prospective register of systematic reviews: CRD42015023448.

\section{Eligibility criteria}

We will select studies based on the criteria outlined below.

\section{Study designs}

The methodological approach advocated by the Cochrane Effective Practice and Organisation of Care (EPOC) group will direct selection of the most robust study designs for the primary analyses. ${ }^{18}$ This is important as it is likely that the majority of available evidence will need to be derived from quasi-experimental and other types of observational studies, which are inherently at risk of bias. ${ }^{10}{ }^{19}$ Following EPOC recommendations, we will therefore restrict our main analyses to the following study designs: randomised controlled trials
(RCTs; including cluster RCTs), controlled clinical trials (CCTs; including cluster CCTs), interrupted time series (ITS studies; including difference-in-difference (DID) designs ${ }^{20}$ ), and controlled before-and-after (CBA) studies. Based on previous experience, ${ }^{10}$ the number of studies in this area that fit the EPOC design criteria is expected to be small. We will therefore, for the primary outcomes, also identify any uncontrolled before-and-after (UBA), prospective or retrospective cohort, case-control and nested case-control studies. For the primary outcomes (see below) we will via sensitivity analyses explore whether the additional inclusion of these study types has any impact on the effect estimates.

\section{Participants}

We will include studies in which the study participants are fetuses (for the outcome 'stillbirth') or children. As our primary interest is in evaluating the impact of tobacco control policies on child health via reducing SHS exposure rather than via reducing active smoking among children, and to also minimise any confounding by active smoking among children, this review will focus on children aged under 12 years. We will, however, include studies that included children up to age 20 years as long as the majority (ie, $>50 \%$ ) of the study population is aged $<12$ years during the at-risk period. Studies that included both adults and children will be eligible for inclusion if paediatric subgroup data are available that fulfil these criteria.

\section{Interventions}

Of interest are population-level and individual-level governmental policies that comply with any of the WHO-advocated tobacco control policies covered in the MPOWER acronym, with the exception of ' $M$ ' for 'Monitor tobacco use and prevention policies'. This is because monitoring, per se, is not expected to improve early-life health outcomes. As categorised according to the (M)POWER acronym, the following policies, instituted at any governmental level (eg, city, state, country), will thus be eligible:

- P (Protect people from tobacco smoke)

- Legislation to create smoke-free public environments (both indoor and outdoor smoke-free policies are of interest)

- O (Offer help to quit tobacco use)

- Tobacco cessation advice or interventions offered through healthcare services

- Free telephone quit lines

- Providing access to free or low-cost cessation medicines

- W (Warn about the dangers of tobacco)

- Health warnings on tobacco products

- Plain packaging of tobacco products

- Mass media campaigns to educate public about dangers of tobacco 
- E (Enforce bans on tobacco advertising, promotion and sponsorship)

- See appendix to Guidelines for implementation of Article 13 of the WHO FCTC for a non-exhaustive list of forms of advertising, promotion and sponsorship within the terms of the FCTC $^{21}$

- R (Raise taxes on tobacco)

- Tax increase on tobacco products

\section{Comparators}

Depending on the study design, eligible comparators include:

- A population living in a geographical area where no change in the policy of interest occurred at the time when the policy was implemented in the geographical area of interest (ie, in the control area the policy has either already been implemented or has not yet been implemented). Examples include (cluster) RCTs and DID studies.

- The population living in the geographical study area of interest before the implementation of the policy of interest in that particular area. This applies to ITS studies, for example.

\section{Outcomes}

We are interested in studies reporting perinatal, infant and paediatric health outcomes that are known to be influenced by antenatal or postnatal tobacco smoke exposure. These are: perinatal, infant and child mortality (including stillbirth), preterm birth, low birth weight, being small for gestational age, congenital anomalies and childhood respiratory disorders (including wheezing/asthma and respiratory infections). According to PRISMA-P guidelines, ${ }^{17}$ further specification of the range of outcomes considered and categorisation into primary and secondary outcomes, is provided in the Outcomes and prioritisation section. Studies reporting at least one primary or secondary outcome in relation to at least one intervention of interest are eligible. Studies evaluating (indicators of) SHS exposure, surrogate outcomes, smoking incidence or behaviour, or economic outcomes only, are not eligible.

\section{Timing}

No restrictions regarding length of follow-up are applicable.

\section{Setting}

There will be no restrictions with regard to study setting.

\section{Language}

No language restrictions will apply. We will seek translation for abstracts published in foreign languages, to assess eligibility as well as for full reports of any potentially eligible studies.
Report type

Articles published in scientific journals, as well as reports in the 'grey literature', will be eligible. For studies reported as abstracts only, authors will be contacted to obtain necessary additional information.

\section{Information sources}

We will search for relevant published studies in the following online data sources: Cochrane Library (CENTRAL), Medline, EMBASE, PsychInfo, Cumulative Index to Nursing and Allied Health Literature (CINAHL), WHO Global Health Library (in addition to MEDLINE covering African Index Medicus (AIM), LILACS, Index Medicus for the Eastern Mediterranean Region (IMEMR), Index Medicus for South-East Asia Region (IMSEAR), Western Pacific Region Index Medicus (WPRIM), WHO Library Database (WHOLIS) and Scientific Electronic Library Online (SciELO)), IndMED, ISI Web of Science, KoreaMed, EconLit, Paediatric Economic Database Evaluation (PEDE), Google Scholar and the ProQuest database of $\mathrm{PhD}$ dissertations. We will search for unpublished and ongoing studies in the overarching WHO International Clinical Trials Registry Platform (ICTRP). Searches will cover the full time period available within each database up until the search date. Although the MPOWER package was introduced by the WHO in 2008, individual tobacco control policies contained in MPOWER have been implemented much earlier in various countries, with evaluation studies of their health impact having been published prior to MPOWER introduction. ${ }^{6} 10$

\section{Search strategy}

We will use a combination of MeSH terms and free text to identify relevant studies from the online data sources. Broad search terms will be used to ensure all relevant studies are identified. The search strategy for MEDLINE is provided as an example in the online supplementary appendix. Specific search strategies will be tailored to the different databases.

We will screen reference lists of articles of interest as well as citations to articles of interest (using Google Scholar) to identify any reports that may have been missed by our search strategy. We will, furthermore, consult an international panel of experts in the field to identify any additional and ongoing studies. This comprehensive approach has previously proved successful in also identifying relevant 'grey literature' (eg, governmental and organisational reports), which is likely to constitute an important share of the pertinent literature on the subject. ${ }^{1022}$

\section{Study records}

\section{Data management}

Records will be entered into an EndNote Library via electronic extraction, or manual input, if necessary. The library will be deduplicated electronically and then checked manually to identify any remaining duplicates. 
Duplicates will be counted for reporting in a PRISMA diagram.

\section{Selection process}

Two reviewers will independently screen titles and abstracts of all unique records identified by the search strategy. Based on consensus, and arbitration by a third reviewer when necessary, records will be selected based on title and abstract for further eligibility screening (ie, reading the full report). Full reports for all selected records will be read and assessed for eligibility independently by two reviewers. Final selection of eligible studies for inclusion in the review will be based on consensus, with arbitration by a third reviewer, if necessary. Should additional information be necessary to assess eligibility of an individual study, we will attempt to obtain such information by contacting the authors. Reasons for exclusion at this stage will be specified and reported in the PRISMA diagram.

\section{Data collection process}

Two reviewers will extract data onto customised data extraction forms. These forms will be piloted using the first five studies identified and amended where necessary. Disagreement regarding extracted data will be resolved by consensus or arbitration by a third reviewer, if necessary. We will contact authors of eligible studies to obtain any relevant information that cannot be retrieved from the original reports.

\section{Data items}

We will extract the following data items from each report: author names, author affiliations, bibliographic information (ie, publication year, journal or book, URL if applicable), study funders, conflicts of interest reported, study type, study site(s), study period(s), description and timing of intervention(s) (including some measure of degree/comprehensiveness), description of comparator, number of clusters and cluster sizes (if relevant), number or participants, treatment group sizes, inclusion/exclusion criteria, participant characteristics (age, gender, socioeconomic status), outcome measures and definitions, data sources used, baseline and postintervention incidence/prevalence of each outcome (for quasi-experimental studies), statistical analysis technique(s) used, unadjusted and adjusted measures of the association between the intervention and outcome including CIs and/or $\mathrm{p}$ values, covariates adjusted for (if applicable), bias assessment, adverse effects, follow-up rate and handling of dropouts. Authors will be contacted to obtain any relevant information that is missing from the reports.

\section{Outcomes and prioritisation}

Primary outcomes are selected based on their recognised associations with SHS exposure and their global relevance to child health, considering prevalence and severity; these are:
- Perinatal mortality (stillbirth+neonatal mortality);

- Preterm birth (live birth at gestational age $<37$ weeks);

- Asthma exacerbations requiring hospital attendance (emergency department visit and/or hospital admission);

- Respiratory tract infections requiring hospital attendance (emergency department visit and/or hospital admission).

Secondary outcomes are:

- Mortality: stillbirth (born dead at gestational age $\geq 24$ weeks), neonatal mortality (death within 28 days), early neonatal mortality (death within 7 days), late neonatal mortality (death between 7 and 28 days), postneonatal mortality (death between 28 days and 1 year), infant mortality (death in the first year of life), child mortality;

- Perinatal outcomes among live births: extremely low birth weight (birth weight $<1000 \mathrm{~g}$ ), very low birth weight (birth weight $<1500 \mathrm{~g}$ ), low birth weight (live birth with birth weight $<2500 \mathrm{~g}$ ), birth weight (continuous scale), small for gestational age (birth weight $<10$ th centile for gestational age), very small for gestational age (birth weight $<3$ rd centile for gestational age), extremely preterm birth (gestational age $<28$ weeks), very preterm birth (gestational age $<32$ weeks), gestational age (continuous scale), congenital anomalies;

- Childhood outcomes: asthma, wheezing, respiratory infections, upper respiratory infections, lower respiratory infections, otitis media with effusion, chronic cough.

We will, from eligible studies that report any of the above outcomes, also extract the following information on evaluation of the potential underlying causal pathways, if provided:

- Changes in maternal (when pregnant) and/or child SHS exposure

- Changes in maternal smoking during pregnancy

- Changes in paternal smoking behaviour

- Changes in child/adult smoking behaviour

Changes in smoking and/or SHS exposure may either be reported or biologically confirmed (preferable; eg, via cotinine measurement in body fluids such as saliva or urine).

\section{Risk of bias of individual studies}

We will assess risk of bias for RCTs, CCTs and CBA studies, using the suggested risk-of-bias criteria for EPOC reviews, ${ }^{23}$ based on the Cochrane Collaboration's tool for assessing risk of bias (Table 8.5.a of the Cochrane Handbook). ${ }^{24}$ For ITS studies we will use EPOC criteria only. ${ }^{23}$ Observational studies will be quality-assessed using the Effective Public Health Practice Project (EPHPP) Quality Assessment Tool for Quantitative Studies. ${ }^{25}$ Risk-of-bias assessment will be performed independently by two reviewers, with any disagreement resolved by consensus or arbitration by a 
third reviewer, if necessary. Risk of bias will then be reported in detailed tabular form.

\section{Data synthesis}

Data will be grouped according to type of intervention and reported in qualitative narrative and tabular form. For each unique combination of an intervention and an outcome of interest, we will extract point estimates and corresponding CIs for effect sizes or association measures, henceforth referred to as effect measures, as appropriate. For dichotomous outcomes, we will extract risk ratios (RR) where possible. We will contact authors to provide RRs if ORs are reported or otherwise estimate these using the formula:

$$
\mathrm{RR}=\frac{\mathrm{OR}}{(1-\mathrm{PEER})+(\mathrm{PEER} \times \mathrm{OR})}
$$

where PEER is the patient-expected event rate in the control group. Unadjusted and adjusted effect measures will both be extracted. When multiple adjusted effect measures for the same combination of an intervention and an outcome are reported, those derived from the most adjusted model will be extracted.

Multiple studies will be combined using meta-analysis when sufficient similarity is present in the intervention under study, in the study population, and in outcome definition, as assessed independently by two reviewers with arbitration of a third reviewer, if necessary. Given anticipated heterogeneity between studies, randomeffects models will be applied in all analyses. For continuous outcomes, we will analyse weighted mean differences, or use standardised mean differences when different measurement scales have been used across studies. Adjusted effect measures will be favoured above unadjusted effect measures for inclusion in meta-analysis. Tax increases are often analysed on a continuous scale when evaluating their impact on health outcomes. ${ }^{13} 15$ We will attempt to standardise the impact estimates from different studies assessing the impact of tobacco taxes so as to facilitate meta-analysis, as appropriate.

Clinical heterogeneity among studies will primarily be assessed qualitatively through identification of differences in study design, inclusion and exclusion criteria, setting, population characteristics, intervention characteristics, outcome definition and ascertainment, and statistical approach. If sufficient clinical homogeneity is considered to be present, $\mathrm{I}^{2}$ test will, in addition, be used to quantitatively assess heterogeneity across studies.

\section{Sensitivity analysis}

Following EPOC guidelines, our primary analyses will be restricted to RCT, CCT, ITS and CBA designs. In order to assess potential bias introduced by this restriction, we will, in a sensitivity analysis, reanalyse the data including findings from any relevant UBA, prospective or retrospective cohort, case-control, and/or nested casecontrol studies on the topic. We will perform additional sensitivity analyses to evaluate the impact of study quality on the association measures, by restricting the analyses to low and moderate risk-of-bias studies.

\section{Subgroup analyses}

In order to inform policy deliberations in the field, we will, where possible, report outcomes according to the comprehensiveness and/or degree of coverage of the intervention (eg, type of environment covered for smoke-free legislation, degree of tobacco tax increase, stop-smoking medicines being free vs low-cost), in subgroup analyses. Furthermore, given the large and increasing global disparities in smoking and smoking-related health outcomes, ${ }^{26} 27$ and the evidence of differential impact of various tobacco control policies on these disparities, ${ }^{11} 152728$ we will, where possible, report the impact of each intervention according to socioeconomic status, alongside describing its overall impact. In an attempt to further minimise potential residual confounding by active smoking among children, we will, where relevant, reanalyse the data after exclusion of studies with active smoking rates $>10 \%$ in the study population.

Meta-analysis will be performed using Stata SE (V.13.0) software.

\section{Meta-bias assessment}

Through our search strategy we will identify any studies for which a protocol was registered but for which results have not been reported, and we will check the study's status with the authors and obtain any data, if possible. For each published study, we will assess whether a protocol was registered prior to undertaking the study. If a protocol exists, we will assess whether any important deviations were present in the study, such as selective outcome reporting. We will construct funnel plots if $>10$ studies are combined in meta-analyses, to assess small study effects that may suggest dissemination bias.

\section{Confidence in cumulative estimate}

On the basis of the combined evaluation of heterogeneity and risk-of-bias assessment across studies, study results, and the results of any meta-analyses and metabias assessments, we will provide an overall interpretation of the strength of the evidence for an association between each intervention and child health outcomes in the Discussion of our report.

\section{ETHICS AND DISSEMINATION}

We will make use of aggregate-level data from prior studies only, for which no ethical assessment is required. We plan to report our study findings according to PRISMA guidelines, ${ }^{29}$ in an appropriate international peer-reviewed scientific journal. We will, in collaboration with public and patient organisations, disseminate our findings to the public. 
Author affiliations

${ }^{1}$ Division of Neonatology, Erasmus University Medical Centre-Sophia Children's Hospital, Rotterdam, The Netherlands

${ }^{2}$ School for Public Health and Primary Care (CAPHRI), Maastricht University Medical Centre, Maastricht, The Netherlands

${ }^{3}$ Centre for Medical Informatics, Usher Institute of Population Health Sciences and Informatics, The University of Edinburgh, Edinburgh, UK

${ }^{4}$ Department of Public Health, Erasmus University Medical Centre, Rotterdam, The Netherlands

${ }^{5}$ Department of Primary Care and Public Health, School of Public Health, Imperial College London, London, UK

${ }^{6}$ Prevention Research Center, Stanford University, Stanford, California, USA

${ }^{7}$ Division of General Internal Medicine and Primary Care, Brigham and Women's Hospital/Harvard Medical School, Boston, Massachusetts, USA

Acknowledgements The authors thank Wichor Bramer for excellent support in developing the search strategy, and Niels Chavannes, Sanne de Josselin de Jong, Juliëtte Kamphuis, Dominique Hamerlijnck and Joke Bottema, for providing feedback on the protocol.

Contributors JVB and AS conceived the study. JVB wrote the first protocol draft. All the authors contributed to development of the methods and writing of the protocol, and have read and approved the final version of the manuscript.

Funding This work was supported by the Erasmus University Medical Centre Research Fellowship, the Netherlands Lung Foundation Junior Researcher Fellowship (grant number 4.2.14.063J0) and Chief Scientist Office (grant number $\mathrm{CZH} / 4 / 1121$ )

Competing interests None declared.

Provenance and peer review Not commissioned; externally peer reviewed.

Open Access This is an Open Access article distributed in accordance with the Creative Commons Attribution Non Commercial (CC BY-NC 4.0) license, which permits others to distribute, remix, adapt, build upon this work noncommercially, and license their derivative works on different terms, provided the original work is properly cited and the use is non-commercial. See: http:// creativecommons.org/licenses/by-nc/4.0/

\section{REFERENCES}

1. World Health Organization. WHO report on the global tobacco epidemic, 2015. Raising taxes on tobacco. 2015. www.who.int/ tobacco/global_report/2015/en

2. Oberg M, Jaakkola MS, Woodward A, et al. Worldwide burden of disease from exposure to second-hand smoke: a retrospective analysis of data from 192 countries. Lancet 2011;377: 139-46.

3. Leonardi-Bee J, Britton J, Venn A. Secondhand smoke and adverse fetal outcomes in nonsmoking pregnant women: a meta-analysis. Pediatrics 2011;127:734-41.

4. Hackshaw A, Rodeck C, Boniface S. Maternal smoking in pregnancy and birth defects: a systematic review based on 173687 malformed cases and 11.7 million controls. Hum Reprod Update 2011;17:589-604

5. Zhang K, Wang X. Maternal smoking and increased risk of sudden infant death syndrome: a meta-analysis. Leg Med (Tokyo) 2013;15:115-21.

6. Tan CE, Glantz SA. Association between smoke-free legislation and hospitalizations for cardiac, cerebrovascular, and respiratory diseases: a meta-analysis. Circulation 2012;126: 2177-83.
7. Dubray J, Schwartz R, Chaiton M, et al. The effect of MPOWER on smoking prevalence. Tob Control 2014. Published Online First: 9 December 2014. doi:10.1136/tobaccocontrol-2014-051834

8. Levy D, Rodriguez-Buno RL, Hu TW, et al. The potential effects of tobacco control in China: projections from the China SimSmoke simulation model. BMJ 2014;348:g1134.

9. Basu S, Glantz S, Bitton A, et al. The effect of tobacco control measures during a period of rising cardiovascular disease risk in India: a mathematical model of myocardial infarction and stroke. PLoS Med 2013;10:e1001480.

10. Been JV, Nurmatov UB, Cox B, et al. Effect of smoke-free legislation on perinatal and child health: a systematic review and meta-analysis. Lancet 2014;383:1549-60.

11. Been JV, Millett C, Lee JT, et al. Smoke-free legislation and childhood hospitalisations for respiratory tract infections. Eur Respir J 2015;46:697-706.

12. Markowitz $\mathrm{S}$. The effectiveness of cigarette regulations in reducing cases of sudden infant death syndrome. J Health Econ 2008;27:106-33.

13. Hawkins SS, Baum CF, Oken E, et al. Associations of tobacco contro policies with birth outcomes. JAMA Pediatr 2014;168:e142365.

14. King C, Markowitz S, Ross H. Tobacco control policies and sudden infant death syndrome in developed nations. Health Econ 2015;24:1042-8.

15. Markowitz S, Adams EK, Dietz PM, et al. Tobacco control policies, birth outcomes, and maternal human capital. J Hum Capital 2013;7:130-60.

16. Been JV, Mackay DF, Millett C, et al. Impact of smoke-free legislation on perinatal and infant mortality: a national quasiexperimental study. Sci Rep 2015;5:13020.

17. Shamseer L, Moher D, Clarke M, et al. Preferred reporting items for systematic review and meta-analysis protocols (PRISMA-P) 2015: elaboration and explanation. BMJ 2015;349:g7647.

18. http://epoc.cochrane.org/sites/epoc.cochrane.org/files/uploads $/ 05 \%$ 20What $\% 20$ study\%20designs $\% 20$ should\%20be\%20included\%20in $\% 20$ an\%20EPOC\%20review\%202013\%2008\%2012 1.pdf

19. Biglan A, Ary D, Wagenaar AC. The value of interrupted time-series experiments for community intervention research. Prev Sci 2000;1:31-49.

20. Dimick JB, Ryan AM. Methods for evaluating changes in health care policy: the difference-in-differences approach. JAMA 2014;312:2401-2.

21. World Health Organization. Guidelines for implementation of Article 13 of the WHO Framework Convention on Tobacco Control (tobacco advertising, promotion and sponsorship), 2008.

22. Been JV, Nurmatov $U$, van Schayck CP, et al. The impact of smoke-free legislation on fetal, infant and child health: a systematic review and meta-analysis protocol. BMJ Open 2013;3: pii: e002261.

23. http://epoc.cochrane.org/sites/epoc.cochrane.org/files/uploads/ Suggested\%20risk\%20of\%20bias\%20criteria\%20for\%20EPOC\% 20reviews.pdf

24. http://handbook.cochrane.org

25. http://www.ephpp.ca/PDF/Quality\%20Assessment\%20Tool_2010_2. pdf

26. Gray R, Bonellie SR, Chalmers J, et al. Contribution of smoking during pregnancy to inequalities in stillbirth and infant death in Scotland 1994-2003: retrospective population based study using hospital maternity records. BMJ 2009;339:b3754.

27. Eikemo TA, Hoffmann R, Kulik MC, et al. How can inequalities in mortality be reduced? A quantitative analysis of 6 risk factors in 21 European populations. PLOS ONE 2014;9:e110952.

28. Hill S, Amos A, Clifford D, et al. Impact of tobacco control interventions on socioeconomic inequalities in smoking: review of the evidence. Tob Control 2014;23:e89-97.

29. Liberati A, Altman DG, Tetzlaff J, et al. The PRISMA statement for reporting systematic reviews and meta-analyses of studies that evaluate healthcare interventions: explanation and elaboration. $B M J$ 2009;339:b2700. 